\section{Qualidade de vida e dimensão ocupacional na esquizofrenia: uma comparação por sexo}

\author{
Quality of life and occupational domain \\ in schizophrenia: a gender comparison
}

\author{
Clareci Silva Cardoso 1,2 \\ Waleska Teixeira Caiaffa 1,2 \\ Marina Bandeira ${ }^{3}$ \\ Arminda Lucia Siqueira 4 \\ Mery Natali Silva Abreu 2,4 \\ José Otávio Penido Fonseca 1,2
}

Departamento de Medicina
Preventiva e Social,
Universidade Federal
de Minas Gerais,
Belo Horizonte, Brasil.
2 Grupo de Pesquisas
em Epidemiologia,
Universidade Federal
de Minas Gerais,
Belo Horizonte, Brasil.
3 Departamento de
Psicologia, Universidade
Federal de São João Del-Rei,
São João Del-Rei, Brasil.
4 Departamento de
Estatística, Universidade
Federal de Minas Gerais,
Belo Horizonte, Brasil.
Correspondência
C. S. Cardoso
Departamento de Medicina
Preventiva e Social,
Faculdade de Medicina,
Universidade Federal
de Minas Gerais.
Av. Alfredo Balena 190,
sala 8.013, Belo Horizonte, MG
30130-100, Brasil.
clarecicardoso@yahoo.com.br

Abstract

Schizophrenia has been associated with low quality of life in patients, and the impact can vary by gender. Knowing gender differences may help implement specific interventions. This study focuses on quality of life in male and female outpatients with schizophrenia, particularly examining the occupational domain. A cross-sectional study using the Quality of Life Scale (QLS-BR) was carried out. Comparisons of scores by gender used uni-and multivariate analyses by means of a classification tree through the CHAID algorithm and ordinal logistic regression. Women showed higher quality of life scores $(p<0.05)$. In the occupational domain, marital status was a relevant variable; single marital status (for men or women) was associated with lower quality of life as compared to married status, with OR $=10.0$ (CI: 2.9-33.3) for men and $O R=4.5$ (CI: 1.2-16.6) for women, respectively. Duration of illness (> 5 years) was another significant factor for lower scores. Women had better quality of life scores than men, suggesting that they have more occupational activities due to their greater participation in domestic and social activities.

Quality of life; Schizophrenia; Gender Identity

\section{Introdução}

A qualidade de vida emergiu nas últimas décadas como um conceito essencial na avaliação do impacto das doenças crônicas, bem como das intervenções terapêuticas, associando-se a indicadores tradicionais como morbidade e mortalidade 1 .

Um dos aspectos fundamentais nas investigações sobre qualidade de vida é a satisfação com o trabalho. Pessoas envolvidas com algum tipo de atividade profissional têm se mostrado mais satisfeitas, o que é verdade para a população geral, para pacientes com esquizofrenia e outras formas de doenças mentais 2,3,4. A participação em uma atividade ocupacional é vista pelos pacientes como uma possibilidade de distração dos sintomas, contribuindo para um melhor estado mental 5 .

A esquizofrenia é considerada uma das mais graves doenças psiquiátricas, que afeta desfavoravelmente a vida dos pacientes em vários domínios. Porém, estudos têm apontado que seus efeitos podem variar de acordo com o sexo 6 , mostrando maior comprometimento nos homens, quando comparado às mulheres 4,6,7,8,9.

A partir dessas descobertas, investigações por sexo na esquizofrenia têm recebido atenção especial. Diferenças foram detectadas quanto à sua distribuição, características, evolução 10 e, mais recentemente, na qualidade de vida 4,6 , $7,8,9,11,12$. A maioria desses estudos indica que 
homens e mulheres possuem diferentes experiências com a esquizofrenia, mas pouco se conhece sobre o reflexo dessas diferenças na percepção do paciente sobre sua qualidade de vida e, principalmente, sobre as suas implicações para a prática clínica e para o planejamento dos serviços de saúde mental 10 .

Vários países utilizam os indicadores de qualidade de vida como ponto central para avaliação da efetividade de tratamentos na saúde mental 1 . No Brasil, estudos de qualidade de vida na esquizofrenia são recentes 13,14 , e seus resultados evidenciam pior qualidade de vida para homens, tanto quando se analisa o escore global de qualidade de vida, quanto no que se refere especificamente ao domínio ocupacional, apontando a necessidade de uma melhor investigação dessas diferenças 9 .

Este conhecimento é particularmente pertinente, considerando que estudos por gênero poderão contribuir para a compreensão do curso da doença e das estratégias de tratamento e planejamento dos serviços de saúde para essa população ${ }^{8}$. No Reino Unido, por exemplo, o planejamento de intervenções é distinto para homens e mulheres 15 .

Este estudo tem como objetivo investigar a qualidade de vida em homens e mulheres com diagnóstico de esquizofrenia, explorando os fatores associados com a baixa qualidade de vida no domínio ocupacional. A qualidade de vida foi definida como a percepção da própria pessoa de seu bem-estar, nível de satisfação e insatisfação com importantes áreas da vida 16, mensurada pela Quality of Life Scale validada para o Brasil (QLS-BR) 13,14.

\section{Método}

\section{Sujeitos}

Participou deste estudo um total de 123 pacientes, incluídos na amostra de acordo com os seguintes critérios: estar desinstitucionalizado; ter diagnóstico confirmado de esquizofrenia (CID-10), com evolução mínima de um ano; estar na faixa etária de 18 e 55 anos; apresentar quadro clínico estável nas três semanas precedentes à coleta de dados. Foram excluídos os pacientes que apresentavam desordem neurológica, retardo mental ou história de dependência de substância química.

\section{Procedimento}

Foi conduzido um estudo transversal, com pacientes selecionados de um Serviço de Refe- rência em Saúde Mental (SERSAM, Divinópolis, Minas Gerais). A investigação foi realizada por meio de entrevistas individuais, conduzidas por psicólogos previamente treinados na condução de entrevistas. Foi aplicado um questionário contendo informações sócio-demográficas e clínicas e a escala de qualidade de vida, QLS-BR. Para a confiabilidade do diagnóstico dos pacientes, $15 \%$ da amostra foram selecionadas de forma aleatória e a história clínica do paciente foi construída com base nos registros do prontuário e encaminhada a dois psiquiatras para avaliação independente. Detalhes metodológicos podem ser encontrados em outras publicações 9,13,14.

A QLS-BR é específica para pacientes com esquizofrenia 17, tendo sido validada para o Brasil 13,14. Possui 21 itens distribuídos em três domínios: (1) social, (2) ocupacional e (3) intrapsíquico e relações interpessoais. Os itens contêm informações sobre sintomatologia e funcionamento do paciente nas três semanas precedentes à entrevista. Cada item é pontuado pelo entrevistador em uma escala do tipo Likert de sete pontos, sendo que os maiores escores refletem uma melhor qualidade de vida 14 .

\section{$\underline{\text { Análise de dados }}$}

Inicialmente homens e mulheres foram comparados usando-se o teste qui-quadrado de Pearson, o teste exato de Fisher ou o teste não-paramétrico de Mann-Whitney, quando apropriado 18 .

Para avaliar a associação entre o escore de qualidade de vida no domínio ocupacional e as características clínicas e sócio-demográficas foi empregada análise multivariada, utilizandose a árvore de decisão por meio do algoritmo CHAID (Chi-squared Automatic Interaction Detector, SPSS Inc., Chicago, Estados Unidos). Este método consiste em sucessivas divisões no conjunto de dados, de forma a torná-lo cada vez mais homogêneo em relação à variável resposta. Posteriormente, os dados foram analisados pela regressão logística ordinal, dentro do modelo de chances proporcionais 19 , possibilitando uma comparação dos resultados nos diferentes métodos.

A variável resposta foi definida como o escore de qualidade de vida codificado em três categorias: qualidade de vida muito comprometida (escore de 0 a 1,99), qualidade de vida comprometida (escore de 2,00 a 4,99 ) e qualidade de vida inalterada (escore de 5,00 a 6,00). As variáveis explicativas foram de natureza sócio-demográfica e clínica, tratadas de forma dicotômica. 
Para o CHAID, todas as variáveis sócio-demográficas e clínicas entraram inicialmente na árvore de regressão. O valor $\mathrm{p} \leq 0,05$ da estatística qui-quadrado usando a correção de Bonferroni foi o principal critério de parada adotado. O procedimento cross-validation utilizando dez subamostras para comparação foi adotado para a validação dos resultados. $\mathrm{O}$ ajuste do modelo final foi avaliado por meio da estatística de risco que compara a diferença entre o valor esperado e o observado pelo modelo, indicando em que medida a árvore corretamente prediz os resultados.

Na regressão logística, o procedimento consistiu em selecionar inicialmente as variáveis que na análise univariada apresentaram valor $\mathrm{p} \leq 0,25$, exceção feita para idade e duração da doença, que entraram no modelo independentemente do valor-p apresentado devido à sua relevância clínica 1,11,20,21,22. A premissa de chances proporcionais foi testada para todas as variáveis individualmente e para o modelo final 19,23.

Ajustou-se o modelo logístico eliminando individualmente as variáveis. Foram considerados como critério para permanência das variáveis no modelo final o valor $\mathrm{p} \leq 0,05$ e a relevância clínica.

Com base nas equações do modelo logístico final foram calculadas as probabilidades de um paciente com determinadas características ser classificado em cada uma das categorias de qualidade de vida. Partindo da primeira equação,

$$
\log \left(\frac{p_{1}}{1-p_{1}}\right)=\alpha_{1}+\beta^{\prime} x
$$

onde $\alpha_{1}$ é a constante e $\beta$ ' o vetor dos coeficientes associados às covariáveis $(\mathrm{x})$, calculouse a probabilidade do paciente ter a qualidade de vida muito comprometida $\left(\mathrm{p}_{1}\right)$. Com base nesta, calculou-se a probabilidade do paciente ter qualidade de vida comprometida $\left(\mathrm{p}_{2}\right)$ por meio da equação

$$
\log \left(\frac{p_{1}+p_{2}}{1-p_{1}-p_{2}}\right)=\alpha_{2}+\beta^{\prime} x
$$

Baseando-se na primeira equação, a categoria qualidade de vida muito comprometida foi comparada com a junção das outras duas categorias (qualidade de vida comprometida + qualidade de vida inalterada), enquanto que na segunda equação, pacientes com algum grau de comprometimento (qualidade de vida muito comprometida + qualidade de vida comprometida) são comparados àqueles com qualidade de vida inalterada. Finalmente a probabilidade do paciente ter qualidade de vida inalte- rada, que é o complementar das probabilidades nas primeiras duas categorias, foi calculada como $1-\left(p_{1}+p_{2}\right)^{24}$.

Foram utilizados os softwares SPSS 11.5, Answertree 3.0 (STATCON, Witzenhausen, Alemanha) e Excel (Microsoft Corporation, Estados Unidos).

O projeto foi aprovado pelo comitê de ética em pesquisa da Universidade Federal de Minas Gerais (UFMG), sob parecer 035/01.

\section{Resultados}

A amostra consistiu de 123 pacientes portadores de esquizofrenia, sendo $74(60,2 \%)$ homens e 49 (39,8\%) mulheres cujas comparações sócio-demográficas e clínicas estão apresentadas na Tabela 1. Na comparação entre sexos, observou-se diferença significativa quanto às seguintes variáveis: idade $(\mathrm{p}=0,02)$, estado civil $(\mathrm{p}=$ $0,03)$, número de pessoas na residência $(p=0,01)$ e com quem o paciente vive $(p<0,01)$. Os homens eram mais jovens, com maior proporção de solteiros, morando com os pais e em residências com maior número de pessoas. Quanto às variáveis clínicas, essa diferença foi detectada para diagnóstico $(\mathrm{p}<0,01)$ e número de medicamentos $(p=0,02)$; maior proporção de homens apresentou o diagnóstico de esquizofrenia paranóide e relatou uso de menor número de medicamentos.

A distribuição dos escores de qualidade de vida por sexo, nos domínios e escala global está apresentada na Tabela 2. No domínio ocupacional, a mediana dos escores de qualidade de vida das mulheres foi de 3,40 comparativamente a 1,62 dos homens $(p<0,01)$. Em todos os cinco itens desse domínio a diferença entre homens e mulheres permaneceu significativa. Vale ressaltar que a diferença nesse domínio refletiu em uma melhor qualidade de vida global para as mulheres, que apresentaram mediana de 2,94, enquanto que os homens apenas $2,17(\mathrm{p}=0,02)$. A mesma diferença foi também observada no item motivação, com mediana de 4,00 para as mulheres e 2,00 para homens ( $p=0,01)$, ou seja, as mulheres se mostraram com maior motivação quando comparadas aos homens.

Nas Figuras 1 e 2 estão apresentados os resultados da análise multivariada por meio da árvore de decisão para homens e mulheres no domínio ocupacional da escala QLS-BR. A análise para as mulheres (Figura 1) mostra o nó raiz com a amostra inteira $(n=49)$, onde $57 \%$ das pacientes estão na categoria da qualidade de vida comprometida, $29 \%$ delas com qualidade de vida muito comprometida e $14 \%$ apre- 
Tabela 1

Características clínicas e sócio-demográficas dos pacientes por sexo e teste de comparação entre os grupos.

\begin{tabular}{|c|c|c|c|}
\hline Características & $\begin{array}{l}\text { Homens } \\
(n=74) \%\end{array}$ & $\begin{array}{l}\text { Mulheres } \\
(n=49) \%\end{array}$ & Valor $p^{*}$ \\
\hline \multicolumn{4}{|l|}{ Sócio-demográficas } \\
\hline \multicolumn{4}{|l|}{ Idade (anos) } \\
\hline$>38$ & 40,5 & 61,2 & 0,02 \\
\hline$<38$ & 59,5 & 38,8 & \\
\hline \multicolumn{4}{|l|}{ Estado civil } \\
\hline Solteiro & 78,4 & 69,4 & 0,03 \\
\hline Casado & 21,6 & 30,6 & \\
\hline \multicolumn{4}{|l|}{ Escolaridade (anos) } \\
\hline$>4$ & 17,6 & 34,7 & 0,261 \\
\hline$<4$ & 82,4 & 65,3 & \\
\hline \multicolumn{4}{|c|}{ Renda familiar (salários mínimos) } \\
\hline$<2$ & 50,7 & 59,2 & 0,35 \\
\hline$>2$ & 49,3 & 40,8 & \\
\hline \multicolumn{4}{|c|}{ Número de pessoas na residência } \\
\hline$<4$ & 59,5 & 81,6 & 0,01 \\
\hline$>4$ & 40,5 & 18,4 & \\
\hline \multicolumn{4}{|l|}{ Com quem vive } \\
\hline Pais & 60,8 & 30,6 & $<0,01$ \\
\hline Outros & 39,2 & 69,4 & \\
\hline \multicolumn{4}{|l|}{ Clínicas } \\
\hline \multicolumn{4}{|l|}{ Diagnóstico (CID-10) } \\
\hline Esquizofrenia paranóide & 94,6 & 77,6 & $<0,01$ \\
\hline Outros tipos & 5,4 & 22,4 & \\
\hline \multicolumn{4}{|l|}{ Duração da doença (anos) } \\
\hline$<5$ & 41,9 & 32,7 & 0,302 \\
\hline$>5$ & 58,1 & 67,3 & \\
\hline \multicolumn{4}{|l|}{ História de internação } \\
\hline Não & 28,4 & 24,5 & 0,634 \\
\hline Sim & 71,6 & 75,5 & \\
\hline \multicolumn{4}{|l|}{ Tratamento atual } \\
\hline Ambulatorial** & 83,8 & 79,6 & 0,553 \\
\hline Outros ${ }^{\star \star \star}$ & 16,2 & 20,4 & \\
\hline \multicolumn{4}{|l|}{ Número de medicamentos } \\
\hline $0-2$ & 51,4 & 30,6 & 0,02 \\
\hline$>2$ & 48,6 & 69,4 & \\
\hline
\end{tabular}

* Qui-quadrado de Pearson ou teste exato de Fisher quando apropriado;

** Tratamento ambulatorial: medicação e consulta periódica:

*** Estão incluídos: sem tratamento algum; acompanhamento ambulatorial, mas sem medicação e uso irregular da medicação. sentaram funcionamento inalterado. A variável que melhor discriminou este grupo foi o estado civil $(p=0,04)$, mostrando a clara separação da árvore.

Para as mulheres solteiras, o medicamento foi importante na árvore independente do número de medicamentos, o maior porcentual delas está na categoria de qualidade de vida comprometida (50 a 67\%). Nesta mesma categoria de qualidade de vida, estão as mulheres casadas e com idade inferior a 38 anos, correspondendo a $60 \%$. Entre as mulheres casadas, acima de 39 anos e duração da doença menor que cinco anos, todas apresentaram qualidade de vida inalterada. Por outro lado, as mulheres casadas, com mais de 39 anos e duração da doença superior a cinco anos, $75 \%$ delas apresentaram qualidade de vida comprometida. O procedimento CHAID mostrou que $61 \%$ das mulheres foram corretamente classificadas (Estimativa de risco $=0,39$ ), enquanto o ajuste obtido por meio da validação cruzada apontou que apenas $39 \%$ delas foram classificadas corretamente (Estimativa de risco $=0,61$ ).

Na Figura 2 estão apresentados os resultados da análise para o sexo masculino. Dos 74 homens, cerca de $62 \%$ apresentaram qualidade de vida muito comprometida, $32 \%$ com qualidade de vida comprometida e apenas $5 \%$ com qualidade de vida inalterada ou funcionamento normal. Assim como para o sexo feminino, a variável que melhor discriminou este grupo foi o estado civil, com uma clara diferença na distribuição das categorias de qualidade de vida. Dos homens casados, $62,5 \%$ apresentaram qualidade de vida comprometida, enquanto que os solteiros com duração da doença acima de cinco anos a maioria tinha qualidade de vida muito comprometida (84\%). Entre homens solteiros com duração da doença de até cinco anos, um menor porcentual apresentou qualidade de vida muito comprometida (59\%). A árvore por meio do CHAID classificou corretamente $70 \%$ dos homens (Estimativa de risco $=0,30$ ) e na validação cruzada houve $64 \%$ de classificações corretas (Estimativa de risco $=0,36$ ).

$\mathrm{Na}$ Tabela 3 estão apresentados os resultados da regressão logística univariada para o domínio ocupacional por sexo. Observa-se que as mesmas variáveis sócio-demográficas preencheram o critério univariado para entrar no modelo (valor $\mathrm{p} \leq 0,25$ ) para homens e mulheres, ou seja, idade, estado civil e com quem vive. Dentre as características clínicas, duração da doença preencheu o critério univariado para o sexo masculino, relato de tratamento médico atual para o sexo feminino e número de medicamentos para ambos. A suposição de chan- 
ces proporcionais foi confirmada para todas as variáveis explicativas individualmente, com pvalor variando de 0,12 a 0,98 .

Os resultados do modelo multivariado estão na Tabela 4. Apenas três variáveis estiveram associadas com baixa qualidade de vida no domínio ocupacional. Para os homens, ser solteiro representa dez vezes mais chances de estar em uma categoria de mais baixa qualidade de vida, quando comparados com os casados. Doença com duração superior a cinco anos também esteve associada à baixa qualidade de vida. Homens com duração da doença maior que cinco anos apresentaram cerca de três vezes mais chances de estar em uma mais baixa categoria de qualidade de vida, quando comparados com aqueles com menor duração da doença.

Entre mulheres, ser solteira apresenta 4,5 vezes mais chances de estar em uma categoria de mais baixa qualidade de vida, quando comparadas com as casadas. O número de medicamentos, apesar de significância limítrofe, indicou que mulheres usando três ou mais medicamentos apresentavam 3,5 vezes mais chances de estar em uma categoria mais baixa de qualidade de vida no domínio ocupacional $(\mathrm{OR}=$ 3,45; IC95\%: 0,94-12,5). A premissa de chances proporcionais foi também atendida para o modelo final, com valor-p entre 0,55 e 0,87.

A probabilidade de baixa qualidade de vida foi calculada com base no modelo logístico final, os resultados são apresentados na Tabela 5. Observou-se que homens solteiros, com duração da doença superior a cinco anos têm probabilidade de 0,82 de estarem em uma categoria de qualidade de vida muito comprometida, enquanto que para homens casados essa probabilidade é de 0,32 . Da mesma forma, mulheres solteiras usando três ou mais medicamentos têm probabilidade de 0,44 de estar na categoria de qualidade de vida muito comprometida, enquanto a chance para uma mulher casada é de 0,15.

De forma geral, para o sexo masculino as duas análises multivariadas apontaram consistentemente as mesmas variáveis associadas a uma mais baixa qualidade de vida: estado civil e duração da doença. Para as mulheres, a árvore de decisão mostrou quatro variáveis: estado civil, idade, número de medicamentos e duração da doença, porém, apenas estado civil e número de medicamentos permaneceram no modelo logístico final.
Tabela 2

Mediana dos escores de qualidade de vida na escala QLS-BR entre homens e mulheres e teste de comparação entre grupos.

\begin{tabular}{|c|c|c|c|}
\hline $\begin{array}{l}\text { Domínios, itens } \\
\text { e escala global }\end{array}$ & $\begin{array}{c}\text { Mediana } \\
\text { dos homens } \\
(n=74)\end{array}$ & $\begin{array}{c}\text { Mediana } \\
\text { das mulheres } \\
(n=49)\end{array}$ & Valor $p^{*}$ \\
\hline Domínio social & 1,86 & 2,57 & 0,10 \\
\hline 1 - Relações familiares & 2,00 & 4,00 & 0,16 \\
\hline 2 - Relações íntimas & 2,00 & 3,00 & 0,99 \\
\hline 3 - Amizades ativas & 2,00 & 2,00 & 0,24 \\
\hline 4 - Atividade social & 2,00 & 2,00 & 0,11 \\
\hline 5 - Rede social & 2,00 & 2,00 & 0,21 \\
\hline 6 - Iniciativa social & 1,00 & 2,00 & 0,21 \\
\hline 7 - Afastamento social & 2,00 & 2,00 & 0,06 \\
\hline Domínio ocupacional & 1,62 & 3,40 & $<0,01$ \\
\hline 9 - Funcionamento ocupacional & 1,00 & 4,00 & $<0,01$ \\
\hline 10 - Nível de realização & 2,00 & 3,00 & $<0,01$ \\
\hline 11 - Subemprego & 1,00 & 3,00 & 0,03 \\
\hline 12 - Satisfação ocupacional & 1,62 & 3,00 & $<0,01$ \\
\hline 17 - Utilização do tempo & 2,00 & 4,00 & $<0,01$ \\
\hline $\begin{array}{l}\text { Domínio intrapsíquico } \\
\text { e relações interpessoais }\end{array}$ & 2,71 & 2,86 & 0,85 \\
\hline 8 - Relações afetivo-sexuais & 1,00 & 1,00 & 0,82 \\
\hline 13 - Sentido de objetivos de vida & 2,00 & 3,00 & 0,36 \\
\hline 15 - Curiosidade & 2,00 & 2,00 & 0,84 \\
\hline 18 - Objetos comuns & 4,00 & 4,00 & 0,88 \\
\hline 19 - Atividades comuns & 2,50 & 3,00 & 0,78 \\
\hline 20 - Empatia & 4,00 & 4,00 & 0,79 \\
\hline 21 - Interação na entrevista & 4,00 & 4,00 & 0,58 \\
\hline Escala global (21 itens) & 2,17 & 2,94 & 0,02 \\
\hline 14 - Motivação & 2,00 & 4,00 & 0,01 \\
\hline 16 - Anedonia & 3,00 & 3,00 & 0,62 \\
\hline
\end{tabular}

* Teste de Mann-Whitney.

\section{Discussão}

Este estudo evidencia diferenças importantes na qualidade de vida entre mulheres e homens, sobretudo no domínio ocupacional. O estado civil apresentou-se como a variável mais relevante para qualidade de vida. Ser solteiro associou-se consistentemente a uma mais baixa qualidade de vida tanto para homens quanto para as mulheres. Maior duração da doença (> 5 anos) foi o segundo marcador em importância principalmente para homens.

Investigações, consistentemente apontam o estado conjugal como preditor de melhor evolução da esquizofrenia. Este marcador é visto como indicador de competência social, repercutindo na qualidade de vida de forma diferen- 
Figura 1

Análise multivariada através da árvore de decisão (CHAID) para as mulheres no domínio ocupacional da escala QLS-BR $(n=49)$.

Domínio ocupacional da QLS-BR

\begin{tabular}{|c|c|c|}
\hline \multicolumn{3}{|c|}{ Nó 0} \\
\hline Categoria & $\%$ & $\mathrm{n}$ \\
\hline $\begin{array}{l}\text { QV muito } \\
\text { comprometida }\end{array}$ & 28,6 & 14 \\
\hline QV comprometida & 57,1 & 28 \\
\hline QV inalterada & 14,3 & 7 \\
\hline Total & $(100,00)$ & 49 \\
\hline
\end{tabular}

Estado civil

valor $p$ ajustado $=0,04$

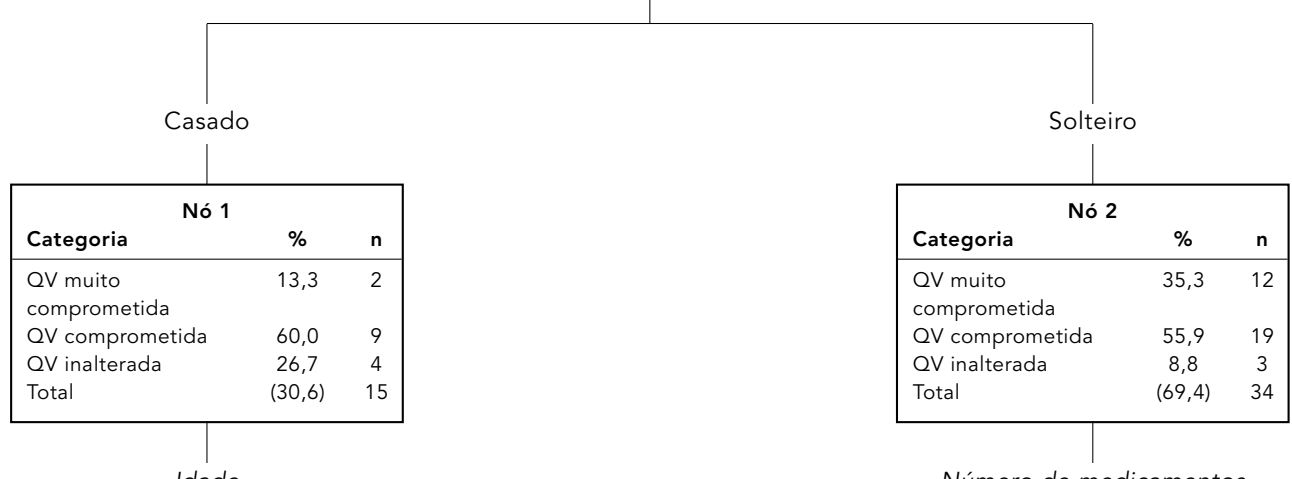

ldade

valor $p$ ajustado $=0,01$

\begin{tabular}{|lcc|}
\cline { 2 - 3 } \multicolumn{1}{l|}{} & \\
\hline \multicolumn{3}{|c|}{ Nenos que 38 anos } \\
\hline Categoria & $\%$ & $\mathbf{n}$ \\
\hline $\begin{array}{l}\text { OV muito } \\
\text { comprometida } \\
\text { OV comprometida } \\
\text { OV inalterada } \\
\text { Total }\end{array}$ & 40,0 & 2 \\
\hline & 0,0 & 3 \\
$(10,2)$ & 5 \\
\hline
\end{tabular}

Número de medicamentos

valor $\mathrm{p}$ ajustado $=0,06$

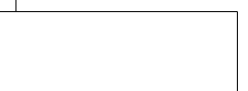

39 anos ou mais

\begin{tabular}{|lcc|}
\hline Categoria & Nó 4 & \\
\hline QV muito & $\%$ & $\mathbf{n}$ \\
comprometida & 0,0 & 0 \\
QV comprometida & 60,0 & 6 \\
QV inalterada & 40,0 & 4 \\
Total & $(20,4)$ & 10 \\
\hline
\end{tabular}

Duração da doença

valor $\mathrm{p}$ ajustado $=0,04$

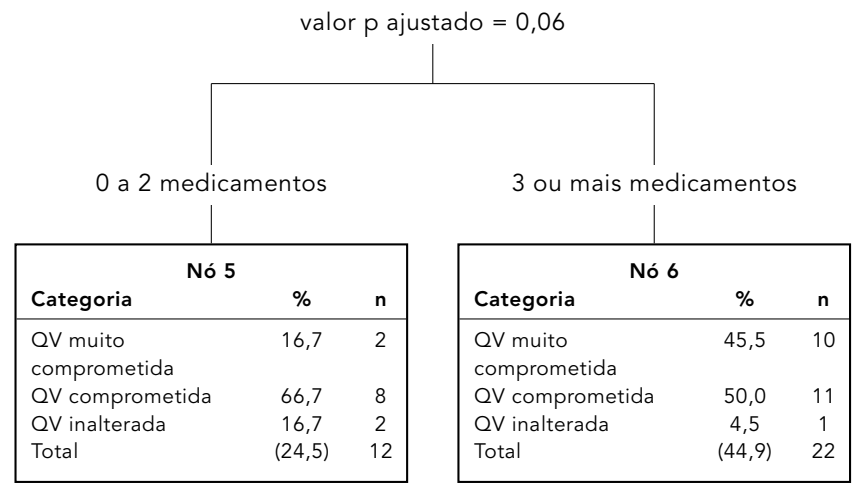

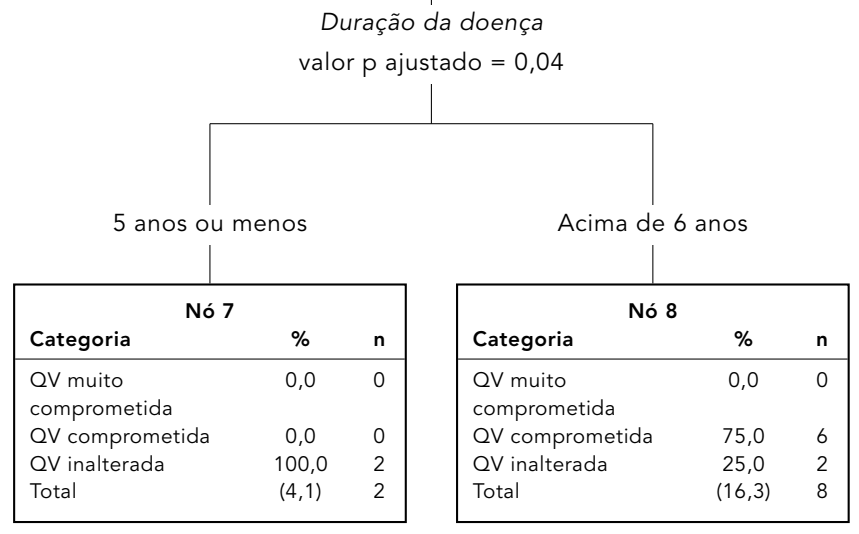

QV = qualidade de vida 
Análise multivariada através da árvore de decisão (CHAID) para os homens no domínio ocupacional da escala QLS-BR $(n=74)$.

Domínio ocupacional da QLS-BR

\begin{tabular}{|c|c|c|}
\hline \multicolumn{3}{|c|}{ Nó 0} \\
\hline Categoria & $\%$ & $\mathrm{n}$ \\
\hline $\begin{array}{l}\text { QV muito } \\
\text { comprometida }\end{array}$ & 62,2 & 46 \\
\hline QV comprometida & 32,4 & 24 \\
\hline QV inalterada & 5,4 & 4 \\
\hline Total & $(100,0)$ & 74 \\
\hline
\end{tabular}

valor $\mathrm{p}$ ajustado $<0,01$

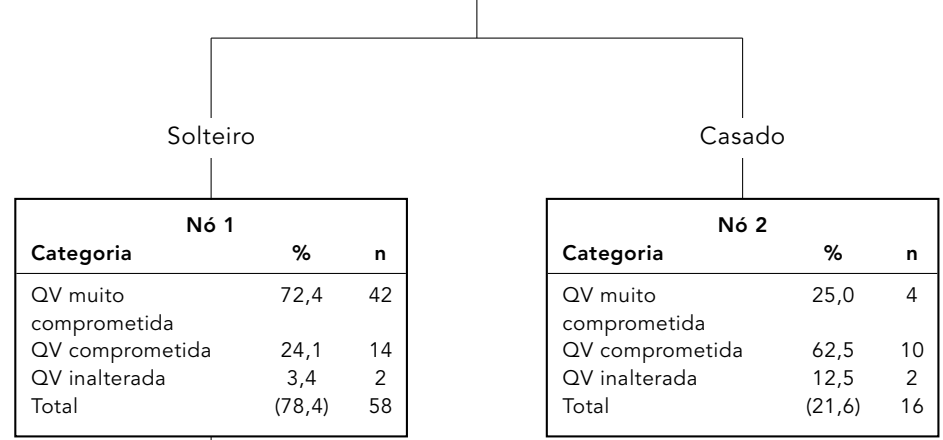

Duração doença

valor $p$ ajustado $=0,02$

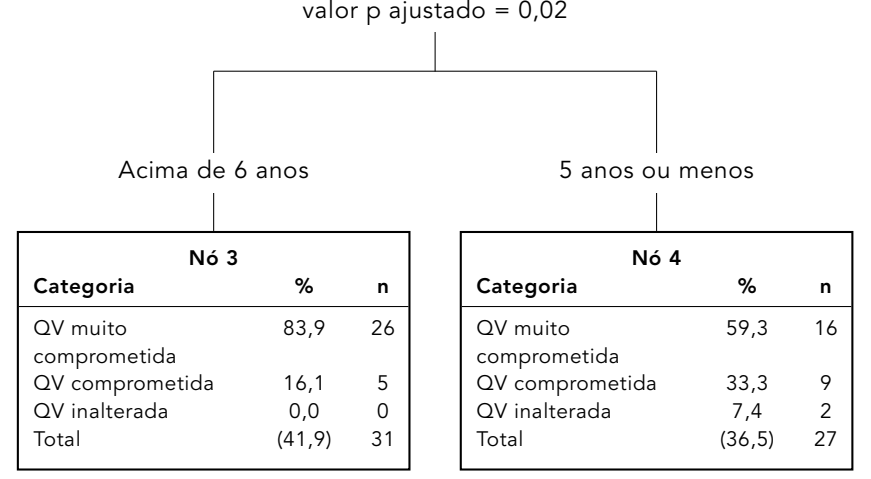

$\mathrm{QV}=$ qualidade de vida

ciada entre os sexos 6 . Neste contexto, as diferenças encontradas entre homens e mulheres em relação ao estado conjugal, com maior magnitude do OR em homens solteiros (OR $=10,0$ versus 4,54 ), pode sugerir que homens com esquizofrenia apresentam maior comprometimento no funcionamento global, associado com maior sintomatologia pré-morbida e início mais precoce da doença 7,21,25. Este quadro, possivelmente, reflete maior prejuízo na com- petência social dos homens devido à expectativa social de que eles devem se mostrar mais ativos no relacionamento com o sexo oposto. Dessa forma, pode ser hipotetizado que, homens com esquizofrenia, em relação às mulheres, apresentam menor capacidade para construir ou manter uma relação. Por outro lado, as mulheres, independente de seu estado conjugal, apresentam-se mais satisfeitas, com melhor integração social, interpessoal e maior envolvi- 
Tabela 3

Regressão logística ordinal univariada* para uma mais baixa qualidade de vida entre homens e mulheres no domínio ocupacional** da escala QLS-BR.

\begin{tabular}{|c|c|c|c|c|}
\hline & \multicolumn{2}{|c|}{$\begin{array}{l}\text { Sexo masculino } \\
(n=74)\end{array}$} & \multicolumn{2}{|c|}{$\begin{array}{l}\text { Sexo feminino } \\
\quad(n=49)\end{array}$} \\
\hline & Odds ratio & Valor $\mathrm{p}$ & Odds ratio & Valor $p$ \\
\hline \multicolumn{5}{|l|}{$\begin{array}{l}\text { Características } \\
\text { sócio-demográficas }\end{array}$} \\
\hline \multicolumn{5}{|l|}{ Idade (anos) } \\
\hline$>38$ & 1,00 & & 1,00 & \\
\hline$<38$ & 2,08 & 0,13 & 2,33 & 0,15 \\
\hline \multicolumn{5}{|l|}{ Escolaridade (anos) } \\
\hline$>4$ & 1,00 & & 1,00 & \\
\hline$<4$ & 1,07 & 0,91 & 1,49 & 0,50 \\
\hline \multicolumn{5}{|l|}{ Estado civil ${ }^{\star \star \star}$} \\
\hline Casado & 1,00 & & 1,00 & \\
\hline Solteiro & 6,87 & $<0,01$ & 3,65 & 0,05 \\
\hline \multicolumn{5}{|c|}{ Renda familiar (salários mínimos) } \\
\hline$>2$ & 1,00 & & 1,00 & \\
\hline$<2$ & 1,41 & 0,47 & 0,76 & 0,63 \\
\hline \multicolumn{5}{|c|}{ Números de pessoas na residência } \\
\hline$>4$ & 1,00 & & 1,00 & \\
\hline$<4$ & 0,70 & 0,47 & 1,74 & 0,45 \\
\hline \multicolumn{5}{|l|}{ Com quem vive $e^{\star \star \star}$} \\
\hline Outros & 1,00 & & 1,00 & \\
\hline Pais & 5,00 & $<0,01$ & 3,30 & 0,06 \\
\hline \multicolumn{5}{|l|}{ Características clínicas } \\
\hline \multicolumn{5}{|l|}{ Diagnóstico (CID-10) } \\
\hline Outros tipos & 1,00 & & 1,00 & \\
\hline Esquizofrenia paranóide & 2,46 & 0,36 & 0,88 & 0,85 \\
\hline \multicolumn{5}{|l|}{ Duração da doença (anos) } \\
\hline$<5$ & 1,00 & & 1,00 & \\
\hline$>5$ & 1,85 & 0,20 & 0,82 & 0,73 \\
\hline \multicolumn{5}{|l|}{ História de Internação } \\
\hline Não & 1,00 & & 1,00 & \\
\hline Sim & 1,65 & 0,33 & 1,22 & 0,76 \\
\hline \multicolumn{5}{|l|}{ Tratamento atual } \\
\hline Outros & 1,00 & & 1,00 & \\
\hline Ambulatorial & 1,82 & 0,33 & 2,59 & 0,18 \\
\hline \multicolumn{5}{|l|}{ Número de medicamentos } \\
\hline $0-2$ & 1,00 & & 1,00 & \\
\hline$>3$ & 0,30 & 0,02 & 2,63 & 0,13 \\
\hline
\end{tabular}

* Teste de chances proporcionais (valor p entre 0,55-0,87);

** Itens do domínio: funcionamento ocupacional, nível de realização, subemprego, satisfação ocupacional e utilização do tempo;

*** Variáveis que apresentaram colinearidade entre si. mento com trabalho, o que certamente favorece sua qualidade de vida $6,8,26,27$.

Da mesma forma, o fato do estado conjugal solteiro estar associado a uma mais baixa qualidade de vida em ambos os sexos, pode apontar para a possível ocorrência de uma menor estimulação social desses pacientes 9 . Neste contexto é possível que homens e mulheres solteiros vivam menos independentemente, em situações de baixa estimulação social, com pouca participação em atividade diária que, por sua vez, encontra-se associada com pior ajustamento funcional na esquizofrenia 6 . Em contraposição, os pacientes casados, talvez favorecidos por seus parceiros, podem apresentar maior estimulação e, conseqüentemente, maior envolvimento social, resultando em melhor qualidade de vida.

Quanto à idade, apesar de resultados controversos na literatura de sua associação com a qualidade de vida 28,29 , nesta investigação, revelou-se importante na análise por meio da árvore de decisão, entre mulheres, sugerindo que as mais novas apresentam pior comprometimento na qualidade de vida. No mesmo sentido, as mulheres que relataram usar três ou mais medicamentos estão também com mais baixa qualidade de vida no domínio ocupacional. Este dado, quando associado ao relato da ocorrência de mal-estar físico, referido como efeito adverso da medicação, sugere uma associação entre estas três variáveis, que por sua vez poderia interferir nas atividades cotidianas das mulheres diferentemente dos homens, que apresentam baixa participação nessas atividades. Da mesma forma, a baixa qualidade de vida em mulheres usando três ou mais medicamentos poderia refletir a ocorrência da síndrome extrapiramidal associada ao uso dos antipsicóticos.

A duração da doença, variável de relevância clínica $11,20,21,22$, associou-se à baixa qualidade de vida para homens em ambas as análises multivariadas e para as mulheres na análise da árvore de decisão, indicando que quanto maior a duração da doença, pior o comprometimento na qualidade de vida. Os dados desta investigação apontam que os homens eram mais novos quando comparados às mulheres, embora a mediana da duração da doença seja a mesma (sete anos) para ambos os sexos. Estes achados apontam para um início mais precoce da doença nos homens, o que é consistente com dados da literatura, quando aponta uma associação entre início mais precoce da doença nos homens e pior evolução da esquizofrenia neste sexo 30.

Os resultados desta investigação nos remetem a um estudo anterior 9 investigando os fa- 
Resultados da regressão logística ordinal multivariada por sexo no domínio ocupacional da escala QLS-BR: estimativa da odds ratio, intervalo de $95 \%$ de confiança, coeficiente, valor $p$, coeficiente das constantes e ajuste do modelo.

\begin{tabular}{|c|c|c|}
\hline Variáveis explicativas (categoria de referência) & Homens $(n=74)$ & Mulheres $(n=49)$ \\
\hline \multicolumn{3}{|l|}{ Variáveis sócio-demográficas } \\
\hline \multicolumn{3}{|l|}{ Estado civil (solteiro) } \\
\hline Odds ratio (IC95\%) & $10,00(2,86-33,33)$ & $4,54(1,20-16,67)$ \\
\hline Coeficiente (valor $p$ ) & $2,30(p<0,01)$ & $1,52(p=0,02)$ \\
\hline \multicolumn{3}{|l|}{ Variáveis clínicas } \\
\hline \multicolumn{3}{|l|}{ Duração da doença (> 5 anos) } \\
\hline Odds ratio (IC95\%) & $3,23(1,10-9,09)$ & \\
\hline Coeficiente (valor $p$ ) & $1,15(p=0,03)$ & - \\
\hline \multicolumn{3}{|l|}{ Número de medicamentos $(>3)$} \\
\hline Odds ratio (IC95\%) & & $3,45(0,94-12,5)$ \\
\hline Coeficiente (valor $p$ ) & - & $1,22(p=0,06)$ \\
\hline \multicolumn{3}{|l|}{ Coeficientes das constantes } \\
\hline Constante 1 (valor $\mathrm{p}$ ) & $0,39(p=0,29)$ & $-1,45(p=0,01)$ \\
\hline Constante 2 (valor $p$ ) & $3,18(p<0,01)$ & $1,65(p<0,01)$ \\
\hline Ajuste do modelo* & 0,925 & 0,941 \\
\hline Teste de chances proporcionais ${ }^{\star \star}$ & 0,878 & 0,558 \\
\hline
\end{tabular}

* Teste de ajuste do modelo (Estatística Deviance);

** Teste de suposição de chances proporcionais (qui-quadrado).

Tabela 5

Probabilidade* de um paciente ser classificado em cada uma das categorias de qualidade de acordo com suas características.

\begin{tabular}{|c|c|c|c|}
\hline Variáveis & $\begin{array}{l}\text { Qualidade de vida } \\
\text { muito comprometida }\end{array}$ & $\begin{array}{l}\text { Qualidade de vida } \\
\text { comprometida }\end{array}$ & $\begin{array}{l}\text { Qualidade de vida } \\
\text { inalterada }\end{array}$ \\
\hline \multicolumn{4}{|c|}{ Duração da doença } \\
\hline \multicolumn{4}{|c|}{ Homem solteiro (anos) } \\
\hline$<5$ & 0,60 & 0,36 & 0,04 \\
\hline$>5$ & 0,82 & 0,16 & 0,01 \\
\hline \multicolumn{4}{|c|}{ Homem casado (anos) } \\
\hline$<5$ & 0,13 & 0,58 & 0,29 \\
\hline$>5$ & 0,32 & 0,56 & 0,12 \\
\hline \multicolumn{4}{|c|}{ Número de medicamentos } \\
\hline \multicolumn{4}{|c|}{ Mulher solteira } \\
\hline $0-2$ & 0,19 & 0,65 & 0,16 \\
\hline$>3$ & 0,44 & 0,50 & 0,05 \\
\hline \multicolumn{4}{|c|}{ Mulher casada } \\
\hline $0-2$ & 0,05 & 0,48 & 0,47 \\
\hline$>3$ & 0,15 & 0,65 & 0,20 \\
\hline
\end{tabular}

* Probabilidades estimadas a partir do modelo logístico ajustado. 
tores associados com a baixa qualidade de vida na esquizofrenia, avaliando a escala global $\mathrm{e}$ todos os domínios da qualidade de vida. Somente duas variáveis estiveram associadas com baixa qualidade de vida no domínio ocupacional: sexo masculino e estado civil solteiro. Homens apresentaram mais chances de baixa qualidade de vida quando comparados às mulheres, assim como os pacientes solteiros. Comparativamente, nesta investigação, trabalhando especificamente a qualidade de vida no domínio ocupacional por sexo e utilizando uma categorização mais ampla da escala QLS-BR, foi possível confirmar o estado civil e observar outras variáveis associadas com uma mais baixa qualidade de vida, tais como número de medicamentos, idade e duração da doença.

A baixa qualidade de vida no domínio ocupacional para homens, conforme sugerida neste estudo, remete à temática ocupacional que aponta para uma estreita relação do trabalho com a satisfação em atividades de lazer e com o sentimento de bem-estar 4,31. Os programas de reabilitação psiquiátrica reforçam a importância da atividade ocupacional, não apenas pelo trabalho em si, mas pelo que ele pode proporcionar ao paciente, tais como habilidade física, independência, relacionamento social e atividades de lazer 32 . Da mesma forma, a ocupação diária, mesmo fora do âmbito do trabalho formal, é vista como importante dimensão para qualidade de vida e para outros aspectos relacionados com a saúde 4 . Dentro desta perspectiva, estreita relação com depressão tem sido encontrada em pacientes desinstitucionalizados, com relato de baixa participação na rede de trabalho. Tais achados são notadamente relevantes para pacientes com esquizofrenia 33 . Apesar de ainda reduzida a participação desses pacientes no trabalho formal, cerca de $15 \%$ para países desenvolvidos 2 , investigações apontam que a presença de alguma atividade ocupacional está marcadamente associada com melhor qualidade de vida, mesmo considerando as divergências culturais 3 .

Mesmo não levando em consideração o trabalho formal e sim a inserção do paciente em atividades ocupacionais diárias, este estudo vem contribuir com informações relevantes dado a escassez de investigações sobre a importância do trabalho na esquizofrenia e, mais raro ainda, estudos que focalizam a ocupação diária 4 . Tal aspecto foi privilegiado no domínio ocupacional da escala utilizada, conforme descrito no processo de adaptação transcultural do instrumento para o contexto brasileiro 13 .

Por fim, nesta investigação é importante ser considerada as limitações de um estudo sec- cional, envolvendo a questão da temporalidade. Neste contexto, não é possível dizer se o fato de permanecer solteiro é uma conseqüência da doença para homens e mulheres ou se o estado civil solteiro seria um fator causal da baixa qualidade de vida. Por outro lado, poderá ainda haver a confusão residual, dado que nem tudo foi mensurado. Este estudo não foi desenhado para testar associações. Por exemplo, não foram incluídas variáveis classicamente associadas com a qualidade de vida, destacamse variáveis relativas ao trabalho formal, presença de depressão, sintomatologia e satisfação com a saúde.

Considerando as análises realizadas, a regressão logística e a árvore de decisão, são técnicas alternativas e complementares, ambas apresentam vantagens e desvantagens. O modelo logístico, método de análise tradicional, é utilizado com bastante freqüência em vários estudos, por meio dele é possível estimar as probabilidades, além de ser um método mais parcimonioso, o que não acontece no CHAID, método mais descritivo e de uso recente na área de saúde, porém, de fácil compreensão.

Os diferentes procedimentos, para os dois métodos, poderiam justificar os diferentes resultados nas análises para as mulheres. Este fato pode ser exemplificado pela seqüência de eliminação das variáveis no modelo logístico para este sexo, dado que, duração da doença e idade, que permaneceram na árvore para as mulheres, foram as últimas variáveis a saírem do modelo logístico.

A inexistência de outros estudos sobre o mesmo tema no Brasil, envolvendo pacientes ambulatoriais, impede a comparação dos resultados aqui encontrados. Quanto à comparação transcultural, esta deve ser conduzida com cautela, uma vez que poderão existir interações complexas de fatores múltiplos nas várias culturas, reforçando a necessidade de pesquisas nesta área 1. Da mesma forma, comparações com outras investigações nos remetem a uma dificuldade inerente ao tema devido à presença de amostras e técnicas de seleção heterogênea, com grande variedade de instrumentos. Muitos estudos falham em descrever adequadamente seus participantes, com exceção do diagnóstico, tornando a comparação difícil, mesmo com uma revisão sistemática da literatura 1,34.

Sabe-se que a esquizofrenia é uma doença característica de adultos jovens, que tem seu início quando os pacientes estão se tornando produtivos, em processo de formação educacional, procurando companheiro e constituindo família. Para muitos, os anos subseqüentes são marcados pelo isolamento social e grande prejuízo 
ocupacional 7,35, assim como é evidenciado neste estudo, principalmente para os homens.

Os serviços de saúde mental devem ser incentivados a identificar estratégias para promover a qualidade de vida dos pacientes de uma forma geral e, mais especificamente, do ponto de vista ocupacional. Este enfoque deve ser direcionado principalmente em relação aos homens, considerando ser a atividade ocupacional, em seu sentido amplo, uma conexão para a integração social. Neste contexto, não se trata apenas das atividades em terapia ocupacional, muitas vezes colocadas pelos serviços de saúde mental como uma imposição ao pa-

\section{Resumo}

A esquizofrenia afeta desfavoravelmente a qualidade de vida dos pacientes, mas seu impacto pode variar de acordo com o sexo. Diferenças relatadas têm orientado o planejamento de intervenções específicas. O objetivo deste estudo foi investigar a qualidade de vida por sexo em pacientes com esquizofrenia, explorando o dominio ocupacional. Foi conduzido um estudo transversal com pacientes em acompanhamento ambulatorial, mensurando a qualidade de vida por meio da Quality of Life Scale (QLS-BR). Comparações dos escores de qualidade de vida foram feitas, seguidas de análises multivariadas usando a árvore de classificação (CHAID) e a regressão logística ordinal. Os resultados apontam melhor qualidade de vida para mulheres $(p<0,05)$. No domínio ocupacional, o estado civil apresentou-se como a variável mais relevante: mulheres e homens solteiros apresentaram baixa qualidade de vida quando comparados com os casados, respectivamente OR = 4,5 (IC95\%: 1,2-16,6) e OR=10,0 (IC95\%: 2,9-33,3). Duração da doença ( $\geq 5$ anos) outro marcador importante, porém no modelo logístico esta variável associou-se à baixa qualidade de vida somente para os homens (OR =3,2; IC95\%: 1,1-9,0). Mulheres apresentaram melhor qualidade de vida, sugerindo maior envolvimento ocupacional, possivelmente resultante de uma maior demanda social e em atividades do lar.

Qualidade de Vida; Esquizofrenia; Identidade de Gênero

\section{Colaboradores}

C. S. Cardoso elaborou o projeto, coordenou os trabalhos, participou do estudo de campo, supervisionou a entrada, a análise e consistência dos dados e redigiu o manuscrito. W. T. Caiaffa participou no desenho do estudo, em todo o processo de campo, auxiliou na entrada e análise de dados. M. Bandeira participou também no desenho do estudo, acompanhou o trabalho de campo e as análises. J. O. P. Fonseca participou na avaliação clínica. A assessoria estatística foi feita por A. L. Siqueira, e M. N. S. Abreu auxiliou na entrada e análise de dados. Todos os autores ajudaram na redação do artigo, aprovando sua versão final. ciente. Trata-se aqui de uma escuta clínica flexibilizada, com programas e estratégias individualizadas, visando a autonomia do paciente e a tão desejada inserção social.

Outra possibilidade poderia ser a verdadeira implementação do acompanhante terapêutico 36 nos serviços de reabilitação, ainda incipiente na prática atual. A proposta do acompanhante terapêutico é de funcionar como mediador entre as estratégias do serviço e as necessidades e dificuldades dos pacientes no cotidiano. Dessa forma, indicadores de qualidade de vida poderiam oferecer subsídios para o planejamento dos serviços, com intervenções orientadas.

\section{Agradecimentos}

Os autores agradecem à Fundação de Amparo à Pesquisa do Estado de Minas Gerais pelo financiamento, processo no CDS-301/02, e também ao Conselho Nacional de Desenvolvimento Científico e Tecnológico e Coordenação de Aperfeiçoamento de Pessoal de Nível Superior pelas bolsas aos pesquisadores: W. T. Caiaffa, M. N. S. Abreu e C. S. Cardoso. Agradecem também ao Grupo de Pesquisa em Epidemiologia, Universidade Federal de Minas Gerais pela estrutura de trabalho. Um agradecimento especial a toda equipe do Serviço de Referência em Saúde Mental de Divinópolis (CERSAM), a todos os pacientes e familiares pelo apoio na produção científica.

\section{Referências}

1. Pinikahana J, Happell B, Hope J, Keks N. Quality of life in schizophrenia: a review of the literature from 1995 to 2000. Int J Ment Health Nurs 2002; 11:103-11.

2. Mueser T, Becker R, Torrey C, Xie H, Bond R, Drake E, et al. Work and nonvocational domains of functioning in persons with severe mental illness: a longitudinal analysis. J Nerv Ment Dis 1997; 185:419-26.

3. Priebe S, Warner R, Hubschid T, Eckele I. Employment, attitudes towards work, and quality of life among people with schizophrenia in three countries. Schizophr Bull 1998; 24:469-77.

4. Eklund M, Hansson L, Bejerholm U. Relationships between satisfaction with occupational factors and health-related variables in schizophrenia outpatients. Soc Psychiatry Psychiatr Epidemiol 2001; 36:79-85.

5. van Dougen CJ. Quality of life and self-esteem in working and nonworking persons with mental illness. Community Ment Health J 1996; 32:535-48.

6. Salokangas R, Honkonen T, Stegard E, Koivisto M. To be or not to be married - that is the question of quality of life in men with schizophrenia. Soc Psychiatry Psychiatr Epidemiol 2001; 36:381-90.

7. Walkup J, Gallagher K. Schizophrenia and the life course: national findings on gender differences 
in disability and service use. Int J Aging Hum Dev 1999; 49:79-105.

8. Roder-Wanner U, Priebe S. Objective and subjective quality of life of first-admitted women and men with schizophrenia. Eur Arch Psychiatry Clin Neurosci 1998; 248:250-8.

9. Cardoso CS, Caiaffa WT, Bandeira M, Siqueira AL, Abreu MA, Fonseca JO. Factors associated with a low quality of life in schizophrenia. Cad Saúde Pública 2005; 21:1338-48.

10. Thornicroft G, Leese M, Tansella M, Howard L, Toulmin H, Herran A, et al. Gender differences in living with schizophrenia. A cross-sectional European multi-site study. Schizophr Res 2002; 57:191200.

11. Moriarty P, Lieber D, Ashley B, White L, Parrella $M$, Harvey P, et al. Gender differences in poor outcome patients with lifelong schizophrenia. Schizophr Bull 2001; 27:103-13.

12. Vandiver VL. Quality of life, gender and schizophrenia: a cross-national survey in Canada, $\mathrm{Cu}$ ba, and U.S.A. Community Ment Health J 1998; 34:501-11.

13. Cardoso CS, Bandeira M, Caiaffa WT, Fonseca JO. Escala de qualidade de vida para pacientes com esquizofrenia - QLS-BR: adaptação transcultural para o Brasil. J Bras Psiquiatr 2002; 51:31-8.

14. Cardoso CS, Caiaffa WT, Bandeira M, Siqueira AL, Fonseca IK, Fonseca JOP. Qualidades psicométricas da escala de qualidade de vida para pacientes com esquizofrenia: Escala QLS-BR. J Bras Psiquiatr 2003; 52:211-22.

15. Department of Health. The National Service Framework for Mental Health. Modern standards and service models. http://www.mentalhealth. org.uk/html/content/updatev02i08.cfm\#3 (acessado em 20/Nov/2004).

16. Becker M, Diamond R, Saifort F. A new patient focused index for measuring quality of life in persons with several and persistent mental illness. Qual Life Res 1993; 2:239-51.

17. Heinrichs DW, Hanlon TE, Carpenter WT. The quality of life scale: an instrument for rating the schizophrenic deficit syndrome. Schizophr Bull 1984; 10:388-98.

18. Medronho RA, Carvalho DM, Bloch KV, Luiz RR, Werneck GL. Epidemiologia. São Paulo: Editora Atheneu; 2003.

19. Hosmer DW, Lemeshow S. Applied logistic regression. $2^{\text {nd }}$ Ed. New York: John Wiley \& Sons; 2000.

20. Menezes PR, Nascimento AF. Curso e prognóstico da esquizofrenia após hospitalização. In: Shirakawa I, Chaves AC, Mari JJ, organizadores. O desafio da esquizofrenia. São Paulo: Lemos Editorial; 1998. p. 67-83.

21. Chaves AC. Diferença entre os sexos na esquizofrenia. In: Shirakawa I, Chaves AC, Mari JJ, organizadores. O desafio da esquizofrenia. São Paulo: Lemos Editorial; 1998. p. 85-101.

22. Chaves AC, Mari JJ, Shirakwa I, organizadores. Gênero, sintomas e desempenho social na esquizofrenia. In: Shirakawa I, Chaves AC, Mari JJ. O desafio da esquizofrenia. São Paulo: Lemos Editorial; 1998. p. 103-17.
23. Lall R, Campbell MJ. A review of ordinal regression models applied on health-related quality of life assessments. Stat Methods Med Res 2002; 11:49-67.

24. Snedker K, Glynn P, Wang C. Ordered/ordinal logistic regression with SAS and STATA. http://staff. Washington.edu/glynn/olr.pdf (acessado em 01/ Dez/2004).

25. Goldstein, JM, Tsuang MT, Faraone SV. Gender and shizophrenia: implications for understanding the heterogeneity of the illness. Psychiatry Res 1989; 28:243-53.

26. Meltzer HY, Burnett S, Bastani B, Ramires IF. Effects of six months of clozapinne treatment on the quality of life of chronic schizophrenia patients. Hosp Community Psychiatry 1990; 41:892-7.

27. Skantze K, Malm U, Dencker SJ, May P, Corrigam P. Comparation of quality of life with standard of living in schizophrenic out-patients. Br J Psychiatry 1992; 161:797-801.

28. Browne S, Roe M, Lane A, Gervin M, Morris M, Kinsella A, et al. Quality of life in schizophrenia: relationship to sociodemographic factors, symptomatology and tardive dyskinesia. Acta Psychiatr Scand 1996; 94:118-24.

29. Corrigan PW, Buican B. The construct validity of subjective quality of life for the several mental ill. J Nerv Ment Dis 1995; 183:281-5.

30. Shepherd M, Watt D, Fallon I, Smeeton N. The natural history of schizophrenia: a five-year follow-up study of outcome and prediction in a representative sample of schizophrenics. Psychol Med Monogra Suppl 1989; 15:1-46.

31. Zemke R, Clark F. Occupational science: the evolving discipline. Philadelphia: F. A. Davis Company; 1996.

32. Holzner B, Kemmber G, Meise V. The impacts of work-related rehabilitation on the quality of life of patients with schizophrenia. Soc Psychiatry Psychiatr Epidemiol 1998; 33:624-31.

33. Cohen CI, Talavera N, Hartung R. Depression among aging persons with schizophrenia. Psychiatr Serv 1996; 47:601-7.

34. Gaite L, Vásquez-Barquero J, Bota C, Ballesteros J, Schene A, Welcher B, et al. Quality of life in patients with schizophrenia in five European countries: The EPSILON study. Acta Psychiatr Scand 2002; 105:283-92.

35. Cohen CI, Sokolovsky J. Schizophrenia and social networks: ex-patients in the inner city. Schizophr Bull 1978; 4:546-60.

36. Bandeira M, Gelinas D, Lesage A. Desinstitucionalização: o programa de acompanhamento intensivo na comunidade. J Bras Psiquiatr 1998; 47:627-40.

Recebido em 24/Mai/2005

Versão final reapresentada em 28/Nov/2005 Aprovado em 11/Dez/2005 